\title{
WHEN TOTAL VARIATION IS ADDITIVE
}

\author{
F. S. CATER
}

\begin{abstract}
Let $f$ and $g$ be continuous functions of bounded variation on $[0,1]$. We use the Dini derivates of $f$ and $g$ to give a necessary and sufficient condition that the equation $V(f+g)=V(f)+V(g)$ holds.
\end{abstract}

Let $f$ and $g$ be continuous functions of bounded variation on [0,1]. We know that $V(f+g) \leqslant V(f)+V(g)$ where $V$ denotes total variation on $[0,1]$. Equality holds in some special cases, for example when $f$ and $g$ are both nondecreasing or both nonincreasing. On the other hand, equality does not hold when $f$ is nonconstant and $g=-f$. In this note we give a necessary and sufficient condition for equality to hold in terms of the four Dini derivates, $D^{+}, D_{+}, D^{-}, D_{-}$, of $f$ and $g$.

DEFINITION 1. We say that $f$ is increasing (or decreasing) at a point $x \in(0,1)$ if

$$
\min \left(D^{+} f(x), D_{+} f(x), D^{-} f(x), D_{-} f(x)\right)>0
$$

(or $\max \left(D^{+} f(x), D_{+} f(x), D^{-} f(x), D_{-} f(x)\right)<0$ ).

We offer the following

THEOREM 1. Let $E_{f}$ denote the set of points where $f$ is increasing and $g$ is decreasing and $E_{g}$ denote the set of points where $g$ is increasing and $f$ is decreasing. Then $a$ necessary and sufficient condition for

$$
V(f+g)=V(f)+V(g)
$$

to hold is that there exist sets $S_{f}$ and $S_{g}$ such that $E_{f} \cup E_{g}=S_{f} \cup S_{g}$ and $\lambda f S_{f}=\lambda g S_{g}$ $=0$, where $\lambda$ is Lebesgue outer measure. Moreover, a necessary condition for (*) to hold is that $\lambda\left(E_{f} \cup E_{g}\right)=0$.

The idea is that $(*)$ holds when there are not "too many" points where one function increases and the other decreases. Before proving Theorem 1, let us discuss some of its consequences. If $f$ is absolutely continuous and $g$ is singular, then $\lambda\left(E_{f} \cup E_{g}\right)=0$ because $g^{\prime}=0$ almost everywhere. Then $\lambda f\left(E_{f} \cup E_{g}\right)=0$ and (*) holds. Thus (*) might hold even when $f$ is strictly increasing and $g$ is strictly decreasing on $[0,1]$. Just make one function absolutely continuous and the other singular. Of course (*) cannot hold if $f$ is increasing and $g$ is decreasing at each point of some subinterval of $[0,1]$.

Received by the editors June 10, 1981.

1980 Mathematics Subject Classification. Primary 26A45, 26A24, 26A30, 26A46.

Key words and phrases. Continuous function of bounded variation, total variation, absolutely continuous function, singular function, Lebesgue outer measure. 
The necessary condition $\lambda\left(E_{f} \cup E_{g}\right)=0$ is not in general sufficient. Consider a singular function $f$ and $g=-f$.

Another familiar example of equation (*) occurs when $f$ and $g$ are the Jordan decomposition of a function of bounded variation, $f+g$, into the sum of two monotone functions.

If $f=f_{1}+f_{2}, g=g_{1}+g_{2}$ where $f_{1}, g_{1}$ are absolutely continuous and $f_{2}, g_{2}$ are singular, then (*) holds for $f$ and $g$ if and only if (*) holds for $f_{1}$ and $g_{1}$ and for $f_{2}$ and $g_{2}$.

When $f$ and $g$ are absolutely continuous, (*) holds if and only if $f^{\prime} g^{\prime} \geqslant 0$ almost everywhere. This also follows from the equation $V(f)=\int_{0}^{1}\left|f^{\prime}\right|$, etc.

We begin with two lemmas. The first is our only result that does not require bounded variation.

LEMMA 1. Let $v$ be a real valued function on $(0,1)$ which is increasing (or decreasing) at each point of a set $S \subset(0,1)$, and let $\lambda v S=0$. Then $\lambda S=0$.

Proof. We prove the case where $v$ is increasing at each point of $S$. We have $S=\cup_{i n k} S_{i n k}$ where

$$
\begin{aligned}
S_{i n k}=\left\{x:(i-1) k^{-1} \leqslant\right. & x<i k^{-1},|v(x)|<n, \\
& \left.(v(x+h)-v(x)) h^{-1}>n^{-1} \text { for }|h|<k^{-1}\right\} .
\end{aligned}
$$

It suffices to prove that each $\lambda S_{i n k}=0$. But $v$ is nondecreasing on $S_{i n k}$. Put

$$
w(x)=\inf \left(\inf v\left([x, 1) \cap S_{i n k}\right), \sup v S_{i n k}\right) .
$$

Then $w$ is nondecreasing on $(0,1)$. Also $w^{\prime} \geqslant n^{-1}$ almost everywhere on $S_{i n k}, w$ is continuous at all but at most countably many points of $S_{i n k}, w S_{i n k}=v S_{i n k}$, and $\lambda w S_{i n k}=0$. It follows from [1, pp. 126, 100] that $\lambda S_{i n k}=0$.

In what follows, $F(x)=V_{0}^{x}(f)$ and $G(x)=V_{0}^{x}(g)$ for $0 \leqslant x \leqslant 1$. Thus $F$ and $G$ are continuous and nondecreasing. Let $f^{*}$ and $F^{*}$ denote the signed Borel measure and the Borel measure induced by the functions $f$ and $F$ respectively. Define $g^{*}$ and $G^{*}$ analogously. For any interval $J$, the diameter of the set $f\left(F^{-1} J\right)$ cannot exceed the length of $J$. Routine arguments show that $\lambda f A \leqslant \lambda F A$ for any set $A \subset(0,1)$. In particular $\lambda f A=0$ if $\lambda F A=0$. We need the converse of this last statement.

Lemma 2. Let $A \subset(0,1)$ such that $\lambda f A=0$. Then $\lambda F A=F^{*} A=0$.

Proof. For any subinterval $(a, b)$ of $(0,1)$ we define the function $s((a, b), y)=$ cardinality of the set $(a, b) \cap f^{-1} y$ for all real $y$. Then from [1, p. 280] we see that $s$ is nonnegative, measurable, summable and

$$
\int s((a, b), y) d y=V_{a}^{b}(f)=F(b)-F(a)=\lambda F(a, b)
$$

In particular, $\int s((0,1), y) d y=V(f)<\infty$. Take any $c>0$. There is an open set $U$ such that $f A \subset U$ and $\int_{U} s((0,1), y) d y<c$. Let $I_{n}, n=1,2,3, \ldots$, be the component 
intervals of the open set $(0,1) \cap f^{-1} U$. Then $\Sigma_{n} s\left(I_{n}, y\right) \leqslant s((0,1), y)$ and

$$
\begin{aligned}
\lambda F\left(\bigcup_{n} I_{n}\right) & =\sum_{n} \lambda F I_{n}=\sum_{n} \int s\left(I_{n}, y\right) d y=\int \sum_{n} s\left(I_{n}, y\right) d y \\
& \leqslant \int_{U} s((0,1), y) d y<c .
\end{aligned}
$$

But $A \subset \cup_{n} I_{n}$, so $\lambda F A<c$. Since $c$ is arbitrary, $\lambda F A=0$. Finally, $F^{*} A=\lambda F A=0$ by $[1$, p. 100].

We are now ready for the proof of Theorem 1.

Proof of SUfFiciency. Assume that $E_{f} \cup E_{g}$ can be covered by two sets $S$ as described. Without loss of generality we assume that the sets $S_{f}$ and $S_{g}$ are Borel sets; note that if $A \supset f S_{f}$ is a $G_{\delta}$-set with $\lambda A=0$, then $f^{-1} A$ is a Borel set and $\lambda f\left(f^{-1} A\right)=0$. Certainly $E_{f}$ and $E_{g}$ are Borel sets because $f$ and $g$ are continuous.

Put

$$
\begin{aligned}
U_{f} & =\left\{x: f \text { is finitely or infinitely differentiable at } x \text { and } f^{\prime}(x)>0\right\}, \\
W_{f} & =\left\{x: f \text { is finitely or infinitely differentiable at } x \text { and } f^{\prime}(x)<0\right\} .
\end{aligned}
$$

Again $U_{f}$ and $W_{f}$ are Borel sets. We define $U_{g}$ and $W_{g}$ analogously for $g$. If $f^{\prime}=0$ everywhere on the Borel set $B$, then $f^{*} B=0\left[1\right.$, p. 127]. Thus $F^{*} B=0$ also. It follows from this and from [1, pp. 126, 127] that $f^{*} \geqslant 0$ on any subset of $U_{f}, f^{*} \leqslant 0$ on any subset of $W_{f}$, and $F^{*}=f^{*}=0$ on any subset of $[0,1] \backslash\left(U_{f} \cup W_{f}\right)$. Likewise $g^{*} \geqslant 0$ on any subset of $U_{g}, g^{*} \leqslant 0$ on any subset of $W_{g}$, and $G^{*}=g^{*}=0$ on any subset of $[0,1] \backslash\left(U_{g} \cup W_{g}\right)$.

We note that $\left(U_{f} \backslash S_{f}\right) \cap\left(W_{g} \backslash S_{g}\right)=\left(U_{f} \cap W_{g}\right) \backslash\left(S_{f} \cup S_{g}\right)=\varnothing$ because $U_{f} \cap$ $W_{g} \subset E_{f}$. Likewise $\left(U_{g} \backslash S_{g}\right) \cap\left(W_{f} \backslash S_{f}\right)=\varnothing$ because $U_{g} \cap W_{f} \subset E_{g}$. Moreover $F^{*} S_{f}$ $=G^{*} S_{g}=f^{*} S_{f}=g^{*} S_{g}=0$ by Lemma 2 . From this it follows that

$$
\begin{aligned}
V(f+g) & \geqslant f^{*}\left(U_{f} \backslash S_{f}\right)-f^{*}\left(W_{f} \backslash S_{f}\right)+g^{*}\left(U_{g} \backslash S_{g}\right)-g^{*}\left(W_{g} \backslash S_{g}\right) \\
& =f^{*} U_{f}-f^{*} W_{f}+g^{*} U_{g}-g^{*} W_{g} \\
& =F^{*}\left(U_{f} \cup W_{f}\right)+G^{*}\left(U_{g} \cup W_{g}\right) \\
& =V(f)+V(g) .
\end{aligned}
$$

ProOF OF NECESSITY. Assume that $V(f+g)=V(f)+V(g)$. Then $V(f+g)=$ $f^{*} U_{f}-f^{*} W_{f}+g^{*} U_{g}-g^{*} W_{g}$, where we use the same notation employed in the proof of sufficiency. Partition $[0,1]$ into the union of two disjoint Borel sets $A$ and $B$ such that the set function $(f+g)^{*}=f^{*}+g^{*}$ is nonnegative on all subsets of $A$ and nonpositive on all subsets of $B$. Fix a Borel set $C \subset U_{f} \cap W_{g} \cap B$. Let $t$ be the nondecreasing function on $[0,1]$ for which $t^{*} D=f^{*} D$ for $D \subset C$ and $t^{*} D=0$ for $D \cap C=\varnothing$. Then $\left(f^{*}-t^{*}\right) D=f^{*} D-f^{*} D=0$ for $D \subset C$, and $f^{*}-t^{*} \geqslant 0$ on subsets of $U_{f}$ and $f^{*}-t^{*} \leqslant 0$ on subsets of $W_{f}$. Also $\left(g^{*}+t^{*}\right) D=g^{*} D+$ $f^{*} D \leqslant 0$ for $D \subset C$ and $g^{*}+t^{*} \leqslant 0$ on subsets of $W_{g}$ and $g^{*}+t^{*} \geqslant 0$ on subsets of $U_{g}$. 
So

$$
\begin{aligned}
V(f+g) & \leqslant V(f-t)+V(g+t) \\
& \leqslant f^{*} U_{f}-t^{*} C-f^{*} W_{f}+g^{*} U_{g}-t^{*} C-g^{*} W_{g} \\
& =f^{*} U_{f}-f^{*} W_{f}+g^{*} U_{g}-g^{*} W_{g}-2 t^{*} C \\
& =V(f)+V(g)-2 f^{*} C=V(f+g)-2 f^{*} C
\end{aligned}
$$

and $f^{*} C=0$. It follows that $F^{*}\left(U_{f} \cap W_{g} \cap B\right)=0$. We can prove in an analogous manner that $G^{*}\left(U_{f} \cap W_{g} \cap A\right)=0$. Let $S_{1}$ be the set formed by adjoining to $U_{f} \cap W_{g} \cap B$ the set of all points where $f$ is not finitely or infinitely differentiable. Likewise $S_{2}$ is formed by adjoining to $U_{f} \cap W_{g} \cap A$ the set of all points where $g$ is not finitely or infinitely differentiable. Then $F^{*} S_{1}=G^{*} S_{2}=0[1$, p. 125], and $E_{f} \subset S_{1} \cup S_{2}$. Likewise there are Borel sets $S_{3}$ and $S_{4}$ such that $F^{*} S_{3}=G^{*} S_{4}=0$ and $E_{g} \subset S_{3} \cup S_{4}$.

Put $T_{f}=S_{1} \cup S_{3}$ and $T_{g}=S_{2} \cup S_{4}$. Then

$$
F^{*} T_{f}=G^{*} T_{g}=0=\lambda F T_{f}=\lambda G T_{g} \quad[1, \text { p. 100]. }
$$

Moreover $\lambda f T_{f}=\lambda g T_{g}=0$, and $E_{f} \cup E_{g} \subset T_{f} \cup T_{g}$. Finally, put $S_{f}=\left(E_{f} \cup E_{g}\right) \cap$ $T_{f}$ and $S_{g}=\left(E_{f} \cup E_{g}\right) \cap T_{g}$. The rest is clear.

To complete the proof of Theorem 1, assume that (*) holds. Then

$$
\lambda\left(S_{f} \cap E_{f}\right)=\lambda\left(S_{f} \cap E_{g}\right)=\lambda\left(S_{g} \cap E_{f}\right)=\lambda\left(S_{g} \cap E_{g}\right)=0=\lambda E_{f}=\lambda E_{g}
$$

by Lemma 1 , and hence $\lambda\left(E_{f} \cup E_{g}\right)=0$.

If $f$ is nondecreasing and $g$ is nonincreasing we can now state when $f$ and $g$ are the Jordan decomposition of the function of bounded variation $f+g$.

COROLLARY 1. Let $f$ be a continuous nondecreasing function and $g$ be a continuous nonincreasing function on $[0,1]$. Then $f$ and $g$ are the Jordan decomposition of the function $f+g$ if and only if there exist sets $S_{f}$ and $S_{g}$ such that $E_{f}=S_{f} \cup S_{g}$ and $\lambda f S_{f}=\lambda g S_{g}=0$.

Proof. Theorem 1.

We find when $F$ and $-G$ are the Jordan decomposition of $F-G$ in terms of the functions $f$ and $g$.

COROllary 2. Let $F, G, f, g$ be as described before. Then

$$
V(f)+V(g)=V(F-G)
$$

holds if and only if

$$
V(f)+V(g)=V(f+g)=V(f-g)
$$

holds.

Proof. Let $E$ be the set of all points where $f$ is increasing or decreasing and $g$ is increasing or decreasing. Let $A$ be the set of all points where $F$ is increasing and $G$ is increasing. Put

$B_{1}=\left\{x: f\right.$ and $F$ are finitely or infinitely differentiable and $\left.\left|f^{\prime}(x)\right|=F^{\prime}(x) \neq 0\right\}$, $B_{2}=\left\{x: g\right.$ and $G$ are finitely or infinitely differentiable and $\left.\left|g^{\prime}(x)\right|=G^{\prime}(x) \neq 0\right\}$. 
By [1, pp. 127, 271],

$$
\lambda f\left([0,1] \backslash B_{1}\right)=\lambda g\left([0,1] \backslash B_{2}\right)=\lambda F\left([0,1] \backslash B_{1}\right)=\lambda G\left([0,1] \backslash B_{2}\right)=0 .
$$

Suppose (***) holds. By Theorem 1 there exist sets $S_{1}$ and $S_{2}$ such that $B_{1} \cap B_{2} \subset$ $S_{1} \cup S_{2}$ and $\lambda f S_{1}=\lambda g S_{2}=0$. By Lemma $2, \lambda F S_{1}=\lambda G S_{2}=0$. Then

$$
\lambda F\left(S_{1} \cup\left(A \backslash B_{1}\right)\right)=\lambda G\left(S_{2} \cup\left(A \backslash B_{2}\right)\right)=0,
$$

and by Theorem $1,(* *)$ holds.

Now suppose (**) holds. By Theorem 1 there exist sets $S_{3}$ and $S_{4}$ such that $B_{1} \cap B_{2} \subset S_{3} \cup S_{4}$ and $\lambda F S_{3}=\lambda G S_{4}=0$. Then $\lambda f S_{3}=\lambda g S_{4}=0$ and

$$
\lambda f\left(S_{3} \cup\left(E \backslash B_{1}\right)\right)=\lambda g\left(S_{4} \cup\left(E \backslash B_{2}\right)\right)=0 .
$$

By Theorem 1, (***) holds.

Corollary 2 can also be proved by other means, but we will not do that here.

\section{REFERENCES}

1. S. Saks, Theory of the integral, 2nd rev. ed., Dover, New York, 1964.

Department of Mathematics, Portland State University, Portland, Oregon 97207 